\title{
Role of I-scan in Diagnosis of Duodenal Mucosal Lesions during Gastroduodenoscopy
}

\author{
Marwa Attia Ibrahim Abdallah ${ }^{1}$, Kamal Ahmed Elkashishy ${ }^{2}$, Samy Eisa \\ Abdelwahab ${ }^{1}$, Nabila Hassan Ahmed ${ }^{1}$,Mohamed Ibrahim Radwan ${ }^{1}$ \\ ${ }^{1}$ Department of Tropical Medicine, Faculty of Medicine, Zagazig University, Egypt. \\ ${ }^{2}$ Department of Pathology, Faculty of Medicine, Zagazig University, Egypt.
}

Corresponding Author Marwa Attia Ibrahim Abdallah

Mobile:

$+201093504338$

E mail:

dr_marwa201041@ya hoo.com

Key words:

I-scan - White light endoscopy - Duodenal mucosal - Celiac Disease
Background and study aim: Duodenal mucosal lesions are usually difficult to diagnose. White light endoscopy(WLE) generally does not visualize the duodenal villous patterns properly and miss small elevated lesions, which result in an inaccurate diagnosis. We aimed to assess the diagnostic accuracy of I-scan technology for evaluation and histological confirmation of mucosal lesions in the duodenum and its role in biopsy targeting.

Patients and Method: This was a single center, cross-sectional study. Patients with any indication for duodenal mucosa histological examination on conventional white light endoscopy WLE and I Scan imaging were included. A definitive diagnosis was determined by histopathology examination of the biopsied specimen.

Results: In our study 41 patients were included (25 males, 16 females, mean age
39.9 years). The criterion-related validity of I-scan as a diagnostic test was $91.9 \%$ sensitivity, $75 \%$ specificity, 97.1\% PPV and $50 \%$ NPV compared to white light endoscopy , which achieved $67.6 \%$ sensitivity, $75 \%$ specificity, 96\% PPV and $20 \%$ NPV. I-scan achieved $95.1 \%$ accuracy, $97 \%$ specificity, $75 \%$ sensitivity in the diagnosis of celiac disease and detection of duodenal villous atrophy compared to WLE, which achieved $92.7 \%$ accuracy, $\quad 100 \%$ specificity, $25 \%$ sensitivity. There was a significant association between disease diagnosis by I-scan and presenting by abdominal pain $(\mathrm{p}<0.026)$.

Conclusion: I-scan represents a simple technique that helps in the diagnosis of duodenal mucosal lesions with high sensitivity, specificity and reduces false negative diagnosis; especially in patients who had abdominal pain.

\section{INTRODUCTION}

Duodenal mucosal lesions are usually difficult to diagnose. White light endoscopy (WLE) commonly does not visualize the duodenal villous patterns properly and miss small raised lesions, which result in imprecise diagnosis [1]. In duodenal lesions the different damage can have a sporadic distribution, and the macroscopic features can be almost related to the degree of the histological lesions [2].

Indeed, at WLE partial villous atrophy may be missed and the normal mucosa appearance at endoscopy does not necessarily mean normal histology. Numerous endoscopic features observed during WLE reveal the villous atrophy presence $[3,4]$. Recently, a biopsy strategy only for patients with villous atrophy detected while using image enhancement techniques, has been proposed [5].

Duodenal carcinoma (DC) is considered a rare gastrointestinal malignancy. Duodenal polyps, either ampullary or non-ampullary adenoma, can be the cause of the development of DC. Some ampullary neoplasms may be early presented with obstructive jaundice or pancreatitis, when a small lesion causes obstruction of ampullary orifice, while nearly all non-ampullary neoplasms are 
incidentally detected through endoscopy for other causes $[\mathbf{6}, \mathbf{7}]$. Nearly all non-ampullary neoplasm presented with gastrointestinal bleeding or bowel obstruction, are usually diagnosed at an advanced stage with bad prognosis [8]. Early detection is needed for improving the survival.

Developed visual endoscopic techniques can detect suspected duodenal mucosal lesions or atrophy; lead to better targeting of the biopsy taking and sensitivity improving of the endoscopy [9]. Various emerging techniques have been tested, alone or in combination, to improve the endoscopist ability to detect mucosal anomalies and to more accurate image the villi architecture, as water immersion, narrow band imaging, I-Scan, high definition-zoom magnification, optical coherence tomography, chromoendoscopy system, and confocal laser endomicroscopy $[\mathbf{1 0}, \mathbf{1 1}]$.

I-scan is Japanese technology settled by Pentax Medical Company for image enhanced by endoscopy. It can be categorized among digital contrast techniques. It permits three distinct image enhancement modalities; they are surface enhancement (SE), contrast enhancement (CE), and tone enhancement (TE). This technology is an imaging post-processing technique, which analyses in real time the endoscopic images without dye practice. It can be achieved just by a button pressing on the endoscope, which it enables the endoscopist to change between the various setting modalities [12].

The aim of this study was to determine the diagnostic accuracy of I-scan technology for evaluation and histological confirmation of mucosal lesions in duodenum and it's role in biopsy targeting in cases admitted to tropical medicine department.

\section{PATIENTS AND METHODS}

\section{I- Patients}

This study was conducted on 41 patients (25 males and 16 females), their ages ranged from 9 to 65years old, who were seen at the Tropical Medicine Department, Faculty of Medicine, Zagazig University Hospitals. Patients were presented with undiagnosed iron deficiency anemia cause, progressive weight loss, chronic diarrhea or persistent abdominal pain. We excluded severe gastric abnormalities, severe hepatic diseases, blood coagulation disorders and failure to complete esophagogastroduodenoscopy.

\section{II- Methods}

All patients were subjected to complete history taking and clinical examination. We stressed on the possible causes of weight loss, dyspepsia, abdominal cramps and chronic diarrhea. We examined the abdomen for epigastric tenderness, organomegally and palpable mass. Laboratory investigations were assessed as complete blood picture, liver function test: alanine amino transferase (ALT), aspartate aminotransferase (AST), serum albumin, total and direct bilirubin by routine enzymatic methods (spinreact), Antitissue transglutaminase (Anti TG-Ig A) and endomysial antibodies (EMA). Pelvi-abdominal Ultrasound was done to detect organomegally, abdominal mass, and exclude chronic liver disease [13]. Upper GIT endoscopy was done using Pentax video endoscopy unit in association with I-Scan techenology imaging with high resolution view. 4 to 6 guided biopsies by I-Scan were taken from the duodenum (in duodenal folding or interfolding) at potential atrophy sites and when disorder was suspected, which were histopathologically examined.

\section{Endoscopic Procedures}

All procedures were done by using I-scan technology included in a Pentax EPK-I processor and EG-2985K. This instrumentation delivers high-resolution images with adjustable digital image magnification over. For this study, we concentrate on the third set of I-scan mode. Patients were sedated with up to $5 \mathrm{mg}$ of midazolam intravenously before undergoing endoscopy. Alternatively, the children were sedated with midazolam $0.1 \mathrm{mg} / \mathrm{kg}$ intravenous administration. All endoscopies were completed by experienced endoscopists. All subjects underwent a careful endoscopic duodenal inspection. The procedure was started with an initial evaluation of the duodenal mucosa by WLE. On the mucosal surface, any debris was removed. In all cases, examination is carried out up to the second part of the duodenum. The features of duodenal mucosal folds, including atrophy, scalloping, and nodularity and any surface lesions such as polyp, nodule, or tumor were determined. Duodenal ampulla was also examined. Then, the endoscope switched to IScan mode. The three I-scan sets, from set 1 to set 3, were used serially, spending an average 
time of $15 \mathrm{~s}$ per single I-scan set. Finally, from the duodenum, directed biopsies were taken.

\section{Histopathology}

Endoscopic biopsies collected in small sterile bottle filled with formalin and transported to the pathological evaluation by expert pathologist within $2 \mathrm{~h}$. Several steps were done to preserve, harden, and protect the tissue from shrinkage. They included Fixation, Dehydration, Clearing, Wax Infiltration, Embedding and Blocks were sectioned at 3-4 micron thickness and were stained with routine hematoxylin and eosin stain.

\section{Statistical analysis}

Social Sciences (SPSS version 20.0)) software Statistical Package for analysis was used. According to the type of data, qualitative data represented as number and percentage and quantitative data represented by mean $\pm \mathrm{SD}$. Sensitivity, specificity \& predictive value were used to assess the validity in the diagnosis. McNemar and Chi-square test (X2) were used to find the association between different variables.

\section{RESULTS}

41 patients were included, the age mean was 39.9 years. The majority were males $61 \%$. Abdominal pain was the main symptom of presented patients $75.6 \%$ with or without other complaints. There was a significant association between disease diagnosis by (I-scan) and abdominal pain ( $\mathrm{p}<0.026) .34 .1 \%$ of patients had normal examination and $46.4 \%$ of them had epigastric tenderness. Laboratory results, including liver function and CBC parameters were normal regarding mean and range, while the $\mathrm{Hb}$ level showed a wide range from 7.5-15.6 $\mathrm{gm} / \mathrm{dl}$. Anaemia was diagnosed in $36.5 \%$ of studied patients, most of cases were microcytic hypochromic $(34.1 \%)$, but there was no significant association between disease diagnosis by (I-scan) and anemia table (1).

Histopathological examination was the gold standard to investigate the taken biopsied specimens, which result the following diagnosis: 5 cases were normal, 3 cases were celiac, one case was eosinophilic gastroenteritis (duodenum and stomach), 29 were chronic duodenitis, 2 were $H$. pylori duodenitis and one case was duodenal bulb adenoma summarized in table (2). $25 \%$ of suspected celiac patients had negative serology, while $75 \%$ of them were anti-TG IgA positive. This table shows that by WLE, $36.6 \%$ of the study patients were normal and $51.3 \%$ had duodenitis while by I-scan $14.6 \%$ were normal and duodenitis appeared in $68.3 \%$ of cases. Duodenitis appeared in the form of superficial ulceration, diffuse mucosal nodularity, severe mucosal congestion and mucosal edema.

Mucosal duodenal lesions in our study patients by I scan viewed as table (3) $48.8 \%$ of the studied patients were normal, 9.7\% were PVA, $2.4 \%$ were polyps, $4.8 \%$ were Ulcers, $14.6 \%$ were Nodules, $14.6 \%$ were fibrous erosion and $4.8 \%$ were abnormal sub mucosal lesions (Accentuated elevation with prominent mucosal architecture with distant mucosal abnormalities), while vascular changes viewed as increased with surface enhancement in $63.4 \%$ of cases fig $(1,2)$.

Overall diagnostic accuracy of I scan was $90.2 \%$ $(95 \% \mathrm{CI})$ and that of WLE was $68.3 \%$ (95\% CI), sensitivity and specificity $91.9 \%$ and $75 \%(95 \%$ CI) for I scan and $67.6 \%$ and $75 \%(95 \% \mathrm{CI})$ for WLE. PPV and NPV to diagnose (or to exclude) were $97.1 \%$ and $50 \%(95 \% \mathrm{CI})$ for I scan and $96.1 \%$ and $20 \%$ (95\% CI) for WLE. Diagnostic accuracy to diagnose celiac for I scan was 95.1 (95\% CI) and that of WLE was $92.7 \%$ (95\% CI), sensitivity and specificity were $75 \%$ and $97 \%$ $(95 \% \mathrm{CI})$ for I scan and $25 \%$ and $100 \%(95 \% \mathrm{CI})$ for WLE table $(4,5)$.

Table (1): The Association between the diagnosis by (I-scan) and clinical picture.

\begin{tabular}{|c|c|c|c|c|c|c|}
\hline \multirow{3}{*}{ Clinical picture } & \multicolumn{4}{|c|}{ Diagnosis (I-scan) } & \multirow{3}{*}{$\chi^{2}$} & \multirow{3}{*}{ P-value } \\
\hline & \multicolumn{2}{|c|}{ Negative } & \multicolumn{2}{|c|}{ Positive } & & \\
\hline & $\mathbf{N}$ & $\%$ & $\mathbf{N}$ & $\%$ & & \\
\hline - $\quad$ Fatigue & 0 & 0 & 1 & 2.4 & 0.18 & 1 \\
\hline - $\quad$ Abdominal pain & 6 & 14.6 & 25 & 60.9 & 5.8 & $0.026 *$ \\
\hline - $\quad$ Dyspepsia & 0 & 0 & 1 & 2.4 & 0.18 & 1 \\
\hline - $\quad$ Diarrhea & 1 & 2.4 & 3 & 7.3 & 0.38 & 0.48 \\
\hline - $\quad$ Pallor & 0 & 0 & 3 & 7.3 & 0.56 & 1 \\
\hline - $\quad$ Melena & 0 & 0 & 1 & 2.4 & 0.18 & 1 \\
\hline
\end{tabular}

Degree of freedom for each $\mathrm{P}$ value equal 1 
Table (2): The histopathological examination of biopsied specimens from the study patients.

\begin{tabular}{|l|c|c|}
\hline \multicolumn{1}{|l|}{} & $\mathbf{N}=\mathbf{4 1}$ & $\mathbf{\% = 1 0 0}$ \\
\hline Pathology: & & \\
- Normal & 5 & 12.2 \\
- Celiac disease & 3 & 7.3 \\
- Eosinophilic gastroenteritis (duodenum and stomach) & 1 & 2.4 \\
- Chronic duodenitis & & \\
- H.pylori duodenitis & 29 & 70.7 \\
- Duodenal bulb adenoma & 2 & 4.8 \\
\hline
\end{tabular}

Table (3): The I-Scan morphological appearance in the patients under study.

\begin{tabular}{|c|c|c|c|}
\hline I-Scan & $\mathrm{N}(\%)$ & & $\mathbf{N}(\%)$ \\
\hline $\begin{array}{ll}\text { Mucosal Lesion: } \\
-\quad \text { Normal } \\
-\quad \text { PVA } \\
-\quad \text { Polyps } \\
-\quad \text { Ulcers } \\
-\quad \text { Nodules } \\
-\quad \text { Fibrinous erosions } \\
-\quad \text { Abnormal sub mucosal lesions: } \\
& \text { Accentuated elevation with } \\
& \text { prominent mucosal architecture } \\
& \text { with distant mucosal abnormalities }\end{array}$ & $\begin{array}{l}20(48.8) \\
4(9.7) \\
1(2.4) \\
2(4.8) \\
6(14.6) \\
6(14.6) \\
2(4.8)\end{array}$ & $\begin{array}{l}\text { Vasculature: } \\
-\quad \text { Normal } \\
\quad-\quad \text { Abnormal: } \\
\text { - Increase with surface } \\
\text { enhancement }\end{array}$ & $\begin{array}{l}15(36.6) \\
26(63.4)\end{array}$ \\
\hline
\end{tabular}

Table (4): The validity of the White light endoscopy and I-Scan as a diagnostic test in the patients under study.

\begin{tabular}{|l|c|c|c|c|c|}
\hline & Sensitivity & Specificity & PPV & NPV & Accuracy \\
\hline White light endoscopy & $67.6 \%$ & $75 \%$ & $96.1 \%$ & $20 \%$ & $68.2 \%$ \\
\hline I Scan & $91.9 \%$ & $75 \%$ & $97.1 \%$ & $50 \%$ & $90.2 \%$ \\
\hline
\end{tabular}

Table (5): Celiac disease lesions diagnosis by the White light endoscopy and I scan in the patients under study.

\begin{tabular}{|l|c|c|c|c|c|}
\hline & Sensitivity & Specificity & PPV & NPV & accuracy \\
\hline White light endoscopy & $25 \%$ & $100 \%$ & $100 \%$ & $92.5 \%$ & $92.7 \%$ \\
\hline I Scan & $75 \%$ & $97 \%$ & $75 \%$ & $97.3 \%$ & $95.1 \%$ \\
\hline
\end{tabular}


Figure (1): (a) Conventional EGD showed slight elevation in the duodenal bulb. (b) I-scan showed accurate detection of mass boundaries with abnormal mucosal architecture and surface breaks.

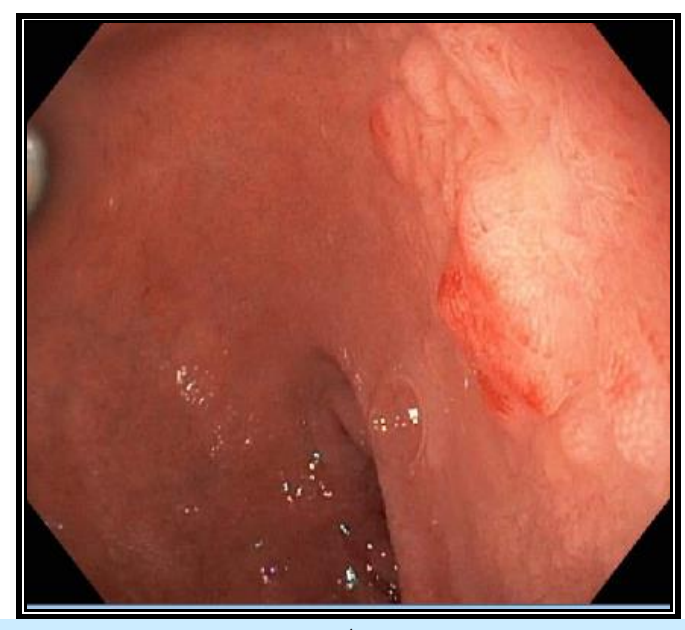

$\boldsymbol{A}$

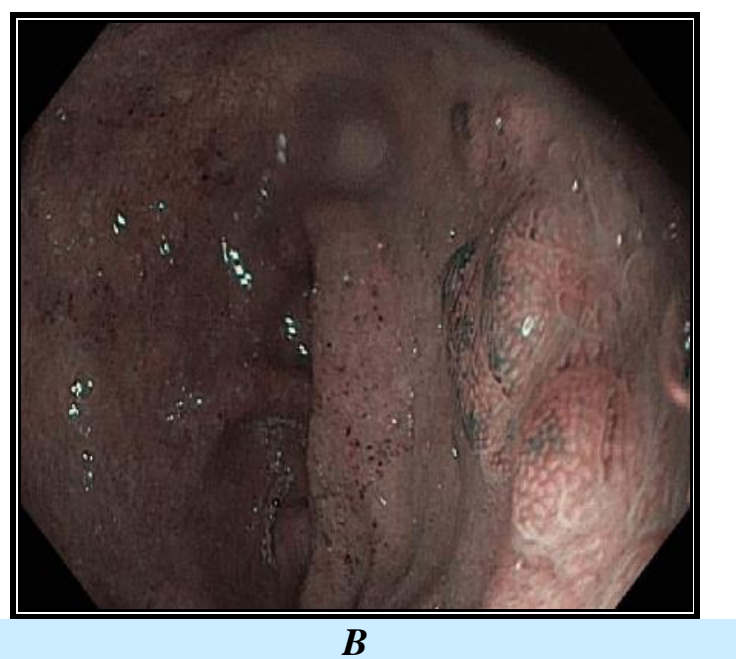

Figure (2): (a) Conventional EGD showed multiple tiny ulcers in the duodenal bulb (b) I-scan showed multiple fibrinous erosion with increased vascular marking and surface enhancement.

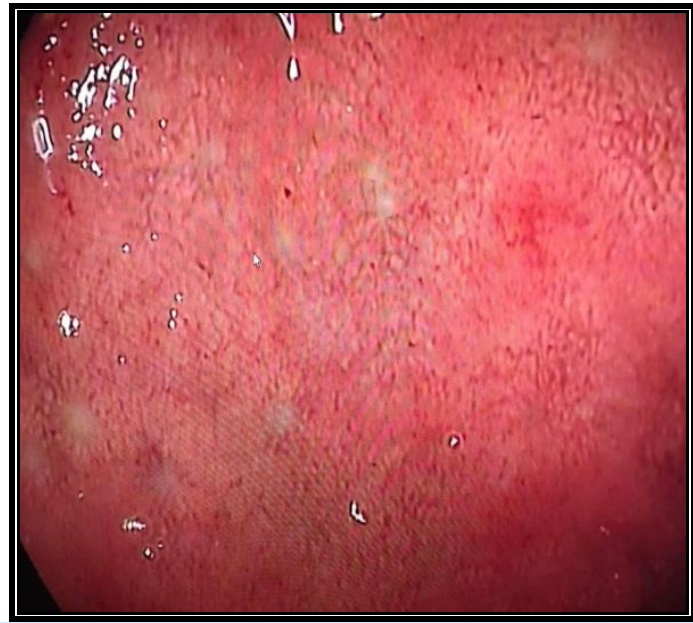

$\boldsymbol{A}$

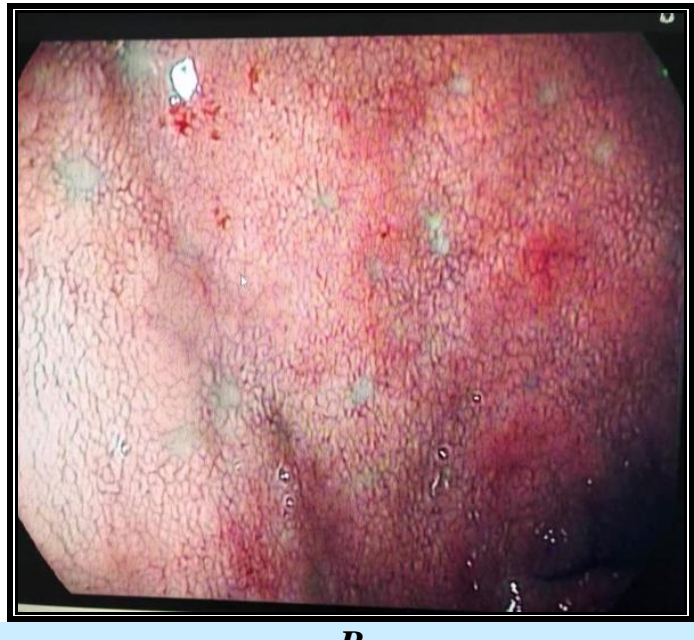

$\boldsymbol{B}$ 
Figure (3): (a) Standard WLE showed continuous and regular mucosa with no mosaic patterns. (b) Iscan showed minor atrophic foci with flattened finger-shaped villi.(c, d)(e,f) duodenal mucosa with poorly distinguishable villi, multifocal active inflammation crypt hyperplasia and lymphocytosishaematoxylin-eosin staining, $(\mathbf{c , d}) \times 100$,scale bar 500um- $(\mathbf{e , f}) \times 400$,scale bar $200 \mathrm{um}$
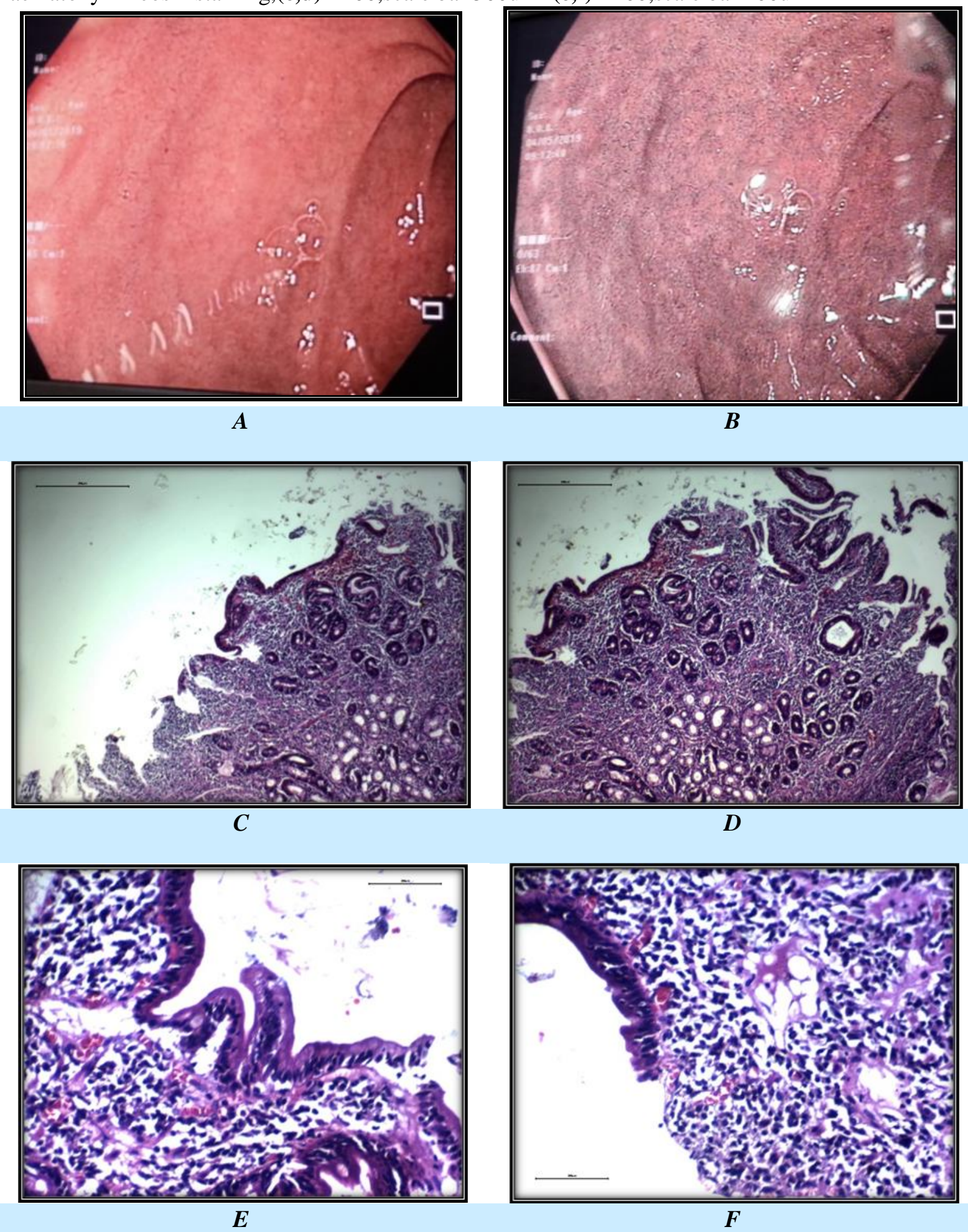


\section{DISCUSSION}

In order to obtain images and enhance image contrast through post-processing algorithms, IScan technology uses various reflective properties for normal and diseased tissues [14]. Without the use of dyes, I-scan enhances the contrast of the mucosal surface and bypasses the problems associated with conventional chromoendoscopy by providing the ability to increase the contrast or 'colour' of the surface in less than a second, just by i-scan button pressing related to the particular feature [15], It can contribute more precise topography of the mucosal surface, and delineate lesion edges by improving vessel and minute mucosal structures [16]. To our knowledge, I-Scan technology has not been considerably tested enough to determine duodenal mucosal lesions.

In our study, 41 patients were included ( 25 male, 16 females, mean age 39.9, range 9-65 years).As regards clinical presentations, abdominal pain was found to be the most common presentation (78\%), while pallor $(7.3 \%)$, diarrhea $(7.3 \%)$, Melena (2.4\%) and Dyspepsia (2.4\%).In our study, we found that there is a significant association between the diagnosis by I-scan and abdominal pain. This was different from Cammarota and et al, who found that out of 115 patients, 32 patients was presented with diarrhea, 31 with abdominal pain, 27 with iron deficiency anemia and 19 patients were presented with weight loss. This difference was due to that our study assessed role of I-scan in all duodenal mucosal lesions while Cammorata assessed the role of I-scan in evaluation of duodenal villous pattern and his study included many patients with diagnosed celiac disease and underwent endoscopy for follow up.

In our study, all routine laboratory investigations were normal, except hemoglobin level show wide variety with range $7.5-15.6 \mathrm{gm} / \mathrm{dl}$. In our study, there was no association between anemia prevalence and diagnosis of duodenal mucosal lesions by I-scan. Our results was conceding with those of Gonen, et al and other study, who studied the diagnostic yield of routine duodenal biopsies in iron deficiency anemia for the diagnosis of celiac disease and the value of duodenal biopsy within routine upper endoscopy, respectively. They found that there was no association between anemia prevalence, and the endoscopic diagnosis of celiac disease or giardiasis $[18,19]$.
Serology evaluation of celiac disease showed positive anti-tissue anti-transglutaminase $\operatorname{IgA}$ antibody and EMA in 3 cases who after that confirmed CD by I- Scan and pathology. Our result was similar to that of Zamani $\mathrm{F}$ and et al, who presented proof that together tTG and EMA should be used for CD diagnosis, although some recommendations suggested that only EMA or tTG is sufficient to diagnose CD patients [20,21]. Schyum and Rumessen also had shown that tTG IgA and DGP IgG have the best clinical diagnostic results, but it is early to endorse a diagnostic approach for $\mathrm{CD}$ in adults that omit duodenal biopsy. In our opinion, use of I-scan targeting biopsies will be of value in such cases [22].

Dutta and Chacko divided I-Scan morphological patterns among the study patients into mucosal and vasculature' changes. As in villous morphology assessment, both surface and vascular features are essential for the evaluation of the neoplastic lesions [23]. Vascular pattern changes and developed angiogenesis are features of neoplastic changes in the tissue. In our study, we use the same classification (mucosal and vascular). The mucosal lesions were distributed as normal, PVA, polyps, ulcers, nodules, fibrous erosion, and abnormal sub mucosal lesions (Accentuated elevation with prominent mucosal architecture with distant mucosal abnormalities), while vasculature changes were classified as normal and abnormal (Increase with surface enhancement).

Our results regarding histopathological examination: $70.7 \%$ of the biopsies were chronic duodenitis, $7.3 \%$ celiac, $4.8 \% \quad H$ pylori duodenitis, $2.4 \%$ for eosinophilic duodenitis and adenoma, while $9.8 \%$ were normal.

I-scan image enhancement technology showed positive endoscopic findings and abnormal pathological changes in 35 patients. I-scan technology resulted in diagnosis of 3 cases of celiac disease, 1 case of esinophilic duodenitis, 1 case of duodenal adenoma, 2 cases of $H$. pylori duodenitis and 29 cases of chronic duodenitis. Iscan in our study was helpful in diagnosis of 2 cases of $H$. pylori duodenitis. Most of the I-scan studies in the gastrointestinal tract focused on the esophagus and colon for Barrett's esophagus evaluation and colorectal polyp detection. Comparatively little information is available on its usefulness in the assessment of duodenal pathology, especially in $H$. pylori. However, 
recent study by Balekuduru and et al., investigated I-scan value in the detection of features similar to $H$. pylori infection. In that study, 68 patients presented with functional dyspepsia examined by M-WLE and I-scan. $H$. pylori infection detection was based on surface vascular features. As a reference gold standard, gastric biopsies were used in detection of $H$. pylori infection, the sensitivity and specificity Iscan were more than $90 \%$ [24].

We have demonstrated a number of patients in which I-scan can be clinically beneficial and affect patient care. It was helpful in diagnosis of a case of duodenal bulb adenoma, who was 42 years old male presented at emergency room by melena, WLE showed slight elevation in duodenal bulb while I-scan showed accurate detection of the mass in duodenal bulb with its boundaries with abnormal mucosal architecture and surface breaks. Testoni and et al studied Iscan in comparison to conventional colonoscopy and they showed higher detection rates of nonprotruding adenomas less than $10 \mathrm{~mm}$ [25]. However, another study evaluated I-scan's ability in small polyps $(<10 \mathrm{~mm}$ ) detection to assist in the accurate assessment of polyp histopathology; they found no significant difference between both techniques in histology prediction.

In our study, the validity of I-scan as a diagnostic test was $90.2 \%$ accuracy $(95 \%$ CI were from $76.87 \%$ to $97.28 \%$ ), $91.9 \%$ sensitivity, $75 \%$ specificity, 97.1\% PPV and 50\% NPV compared to WLE, which achieved $68.2 \%$ accuracy $(95 \%$ CI were from $51.91 \%$ to $81.92 \%$ ), $67.6 \%$ sensitivity, $75 \%$ specificity, 96\% PPV and $20 \%$ NPV. Our result was in agreement with Hancock and et al who studied the uses of I-scan and concluded that it helped in the diagnosis of neoplastic changes as Barrett's esophagus, gastric lymphoma, duodenal adenoma and lymphoma [16].

In our study, I-scan achieved $95.1 \%$ accuracy, $97 \%$ specificity, $75 \%$ sensitivity in the diagnosis of celiac disease and detection of duodenal villous atrophy compared to WLE that achieved $92.7 \%$ accuracy, $100 \%$ specificity, $25 \%$ sensitivity. In WLE this may be due to poor sensitivity of mucosal fold changes such as atrophy, scalloping and nodularity compared to histology and similar cause was concluded by Banerjee and Reddy [27]. Our results were closely near the findings of Cammarota and et al [17], who reported that I-scan achieved excellent outcomes in the depiction of duodenal villous patterns, and reached $100 \%$ accuracy in diagnosis marked villous atrophy, 90\% accuracy, $77 \%$ sensitivity and $94 \%$ specificity in (PVA pattern) in celiac disease.

\section{CONCLUSION}

Our findings show that I-scan represents a simple technique that help in the diagnosis of duodenal mucosal lesions with sensitivity $91.9 \%$, specificity $75 \%$ and accuracy $90.2 \%$ by providing detailed mucosal topography especially in patients presented by abdominal pain, which can directly impact endoscopic management and importantly improve biopsy sampling so, potentially reducing the need for blind biopsies and false-negative cases.

\section{Declaration of conflicting interests: None}

\section{Funding: None}

Ethical approval: Written informed consent was obtained from all patients and IRB approval.

\section{REFERENCES}

1. Oxentenko A, Grisolano S, Murray J, Burgart L, Dierkhising R, Alexander J. The insensitivity of endoscopic markers in celiac disease. The American journal of gastroenterology 2002; 97(4): 933-938.

2. Dickey W, McMillan A, Hughes F. Sensitivity of serum tissue transglutaminase antibodies for endomysial antibody positive and negative coeliac disease. Scandinavian Journal of Gastroenterology 2001; 36(5):511-514.

3. Mauriño E, Capizzano H, Niveloni S, Kogan Z, Valero J, Boerr L et al. Value of endoscopic markers in celiac disease. Digestive Diseases and Science 1993; 38(11):2028-2033.

4. Bardella M, Minoli G, Radaelli F, Quatrini M, Bianchi P , Conte D. Gastrointestinal Endoscopy 2000; 51(6):714-716.

5. Iacucci M, Ghosh S. Routine duodenal biopsies to diagnose celiac disease. Canadian Journal of Gastroenterology 2013; 27(7):385

6. Guzzardo G, Kleinman M, Krackov J , Schwartz S. Recurrent acute pancreatitis caused by ampullary villous adenoma. Journal of Clinical Gastroenterology 1990;12 (2):200-202.

7. Jean M , Dua K. Tumors of the ampulla of Vater. Current Gastroenterology Reports 2003; 5: $171-175$. 
8. Chini P, Draganov P. Diagnosis and management of ampullary adenoma: The expanding role of endoscopy. World Journal of Gastrointestinal Endoscopy 2011;3:241-247.

9. Lo A, Guelrud M, Essenfeld H, Bonis P. Classification of villous atrophy with enhanced magnification endoscopy in patients with celiac disease and tropical sprue. Gastrointestinal Endoscopy 2007; 66(2):377-382.

10. Oxentenko A , Murray J. Celiac disease: ten things that every gastroenterologist should know. Clinical Gastroenterology\& Hepatology 2015; 13: 1396-1404.

11. Coda S, Thillainayagam, A. The state of the art in advance endoscopic imaging for the detection and evaluation of dysplasia and early cancer of the gastrointestinal tract. Clinical and Experimental Gastroenterology 2014; 7:133150.

12. Kodashima $\mathrm{S}$, Fujishiro $\mathrm{M}$. Novel imageenhanced endoscopy with i-scan technology. World journal of gastroenterology 2010; 16(9), 1043.

13. Migaleddu V, Quaia E, Scano D, Virgilio G. Inflammatory activity in Crohn disease: ultrasound findings. Abdominal imaging 2008; 33(5), 589.

14. Li H, Hou X, Lin R, Fan M, Pang S, Jiang, L et al. Advanced endoscopic methods in gastrointestinal diseases: a systematic review. Quantitative Imaging in Medicine and Surgery 2019; 9(5), 905.

15. Hoffman A, Sar F, Goetz M, Tresch A, Mudter J, Biesterfeld $S$ et al. High definition colonoscopy combined with i-Scan is superior in the detection of colorectal neoplasias compared with standard video colonoscopy: a prospective randomized controlled trial. Endoscopy 2010; 42(10), 827-833.

16. Hancock S, Bowman E, Prabakaran J, Benson M, Agni R , Pfau P. Use of i-scan endoscopic image enhancement technology in clinical practice to assist in diagnostic and therapeutic endoscopy: a case series and review of the literature. Diagnostic and therapeutic endoscopy 2012.

17. Cammarota G, Ianiro G, Sparano L, La Mura R, Ricci R, Larocca $M$ et al. Image-enhanced endoscopy with I-scan technology for the evaluation of duodenal villous patterns. Digestive diseases and sciences 2013; 58(5), 1287-1292.
18. Gonen C, Yilmaz N, Yalcin M, Simsek I , Gonen O. Diagnostic yield of routine duodenal biopsies in iron deficiency anaemia: a study from Western Anatolia. European Journal of Gastroenterology\& Hepatology 2007;19(1):3741.

19. Tischendorf J, Wopp K, Streetz L, Bach J, Meyer M , Tischendorf S . Die Wertigkeit der tiefen Duodenal biopsie im Rahmen der Routineendoskopie: Eine prospektive Studie mit 1000 Patienten [The value of duodenal biopsy within routine upper endoscopy: a prospective study in 1000 patients].Zeitchrift for Gastroenterology 2008; 46(8):771-775.

20. Zamani F, Mohamadnejad M, Shakeri R, Amiri A, Najafi S, Alimohamadi $M$ et al. Gluten sensitive enteropathy in patients with iron deficiency anemia of unknown origin. World journal of gastroenterology 2008; 14(48), 7381-7385.

21. Hill D. What are the sensitivity and specificity of serologic tests for celiac disease? Do sensitivity and specificity vary in different populations? Gastroenterology 2005;128,S25-S32.

22. Schyum C, Rumessen J. Serological testing for celiac disease in adults. United European gastroenterology journal 2013; 1(5), 319-325.

23. Dutta K , Chacko A. Emerging role of narrow band imaging in duodenum. World Journal of Gastrointestinal Endoscopy 2015; 7(16), 1216.

24. Balekuduru B, Rau A, Gouri M, Shetty A, Subbaraj B. Magnifying i-scan imaging for endoscopic diagnosis of helicobacter pylori infection: a prospective study. Journal of Digestive Endoscopy 2020; 11(02), 112-117.

25. Testoni A, Notaristefano C, Vailati C, Di Leo M , Viale E . High-definition colonoscopy with iScan: better diagnosis for small polyps and flat adenomas. World journal of gastroenterology 2012; 18(37), 5231.

26. Bouwens $\mathrm{W}$, de Ridder $\mathrm{R}$, Masclee A, Driessen A, Riedl G, Winkens B et al . Optical diagnosis of colorectal polyps using highdefinition i-scan: an educational experience. World journal of gastroenterology 2013; 19(27), 4334-4343.

27. Banerjee R, Reddy N. High-resolution narrowband imaging can identify patchy atrophy in celiac disease: targeted biopsy can increase diagnostic yield. Gastrointestinal endoscopy 2009; 69(4), 984-985. 